\title{
Intervention treatment on massive hemoptysis of pulmonary aspergilloma
}

\author{
GUANGHUI HE ${ }^{1}$, WENYU LIU ${ }^{2}$, ZHIQIANG GAO ${ }^{3}$, ZHI GAO $^{1}$, \\ HONGSHENG GAO $^{1}$ and YANJIE WANG ${ }^{1}$
}

\author{
Departments of ${ }^{1}$ Interventional Therapy, ${ }^{2}$ Tuberculosis and ${ }^{3}$ Pharmacy, \\ The Second People's Hospital of Weifang, Weifang, Shandong 261041, P.R. China
}

Received May 31, 2016; Accepted February 24, 2017

DOI: $10.3892 /$ etm.2017.4230

\begin{abstract}
Retrospective analysis was carried out on intervention treatment and the effects of massive hemoptysis of pulmonary aspergilloma. Twenty-five cases diagnosed as massive hemoptysis of pulmonary aspergilloma were performed with imaging of bronchial arteries and other related blood vessels in order to assess blood vessel type and the number involved in the disease and perform embolotherapy on the offending artery. There were 68 bleeding arteries in 25 cases, of which there were 36 bronchial arteries (52.94\%), 15 intercostal arteries (22.06\%), 9 internal thoracic arteries (16.17\%), 5 inferior phrenic arteries $(7.35 \%)$, and 3 pulmonary arterial branches $(4.41 \%)$ and all of them were in embolism condition. For 25 cases, 21 bleeding cases were stopped immediately $(84.00 \%), 3$ bleeding cases were stopped after the 2nd embolism, and 1 case had a small amount of hemoptysis after surgery discontinuously, which was stopped after corresponding treatment with no severe complications. Ten cases used polyvinyl alcohol (PVA) particulate embolization solely, 12 cases used PVA with spring coil and 3 cases used PVA, spring coil and $N$-butyl cyanoacrylate (NBCA) glue. In conclusion, patients who suffer from massive hemoptysis of pulmonary aspergilloma and whose medical treatments are not effective and who are not willing to receive surgical removal, intervention treatment is an effective method. PVA, spring coil and NBCA glue can be effectively used in hemoptysis embolism and pulmonary artery embolism can be considered when systemic embolism is not effective.
\end{abstract}

\section{Introduction}

Pulmonary aspergillosis is an infectious disease caused by Aspergillus and one of its special types is pulmonary aspergilloma, most of which is secondary to pulmonary tuberculosis. Its

Correspondence to: Dr Hongsheng Gao, Department of Interventional Therapy, The Second People's Hospital of Weifang, 7 Xiaoyuan Street, Weifang, Shandong 261041, P.R. China E-mail: gao_hongsheng9@163.com

Key words: pulmonary aspergilloma, hemoptysis, intervention treatment main clinical symptoms are repeated hemoptysis and features are repetition, deferment and different amounts of hemoptysis, in most cases. General symptoms are slight but some patients have unmanageable massive hemoptysis (hemoptysis amount $>500 \mathrm{ml} /$ day or hemoptysis amount at one time $>300 \mathrm{ml}$ ) (1), indicating rare clinical acute lung disease, for which medical hemostatic drugs are not effective.

Currently, most Chinese scholars insist on surgery, which has achieved good results $(2,3)$, but some patients are not willing to receive surgery or cannot withstand surgery. To explore the intervention embolotherapy effects, a retrospective analysis was performed on the intervention treatment of 25 massive hemoptysis of pulmonary aspergilloma cases. The study is presented in detail in the following sections.

\section{Materials and methods}

General materials. Twenty-five massive hemoptysis of pulmonary aspergilloma cases diagnosed in the Second People's Hospital of Weifang (Shandong, China) from 2008 to 2016 were chosen as study subjects. Of these 25 cases, 11 were males and 4 females, aged 20-78 years and an average of $56.5 \pm 5.2$ years. Location of aspergilloma: 12 cases on superior lobe of the right lung, 3 cases on inferior lobe of the right lung, 8 cases on superior lobe of the left lung, and 2 cases on inferior lobe of the left lung. The diameter of focus was $2-8 \mathrm{~cm}$. Sputum smear, or sputum culture was performed before surgery, and 3 cases were positive lung aspergillosis. Chest computed tomography (CT) showed 10 cases with representative massive shadow with 'meniscus sign' (Fig. 1), 5 cases received skin puncture lung biopsy, and 7 cases received bronchoscopic biopsy. This study was approved by the Ethics Committee of the Second People's Hospital of Weifang. Signed written informed consents were obtained from all participants before the study.

Clinical manifestation. With hemoptysis as the main clinical symptom which repeatedly occurs and lasts for a long time and cannot be treated through conventional medical treatment, the course of disease is long for most patients and their nutrition condition is poor, with pale appearance, low immunity and general symptoms such as fever, yellow sputum, and weakness if complicated with bacterial infection. 

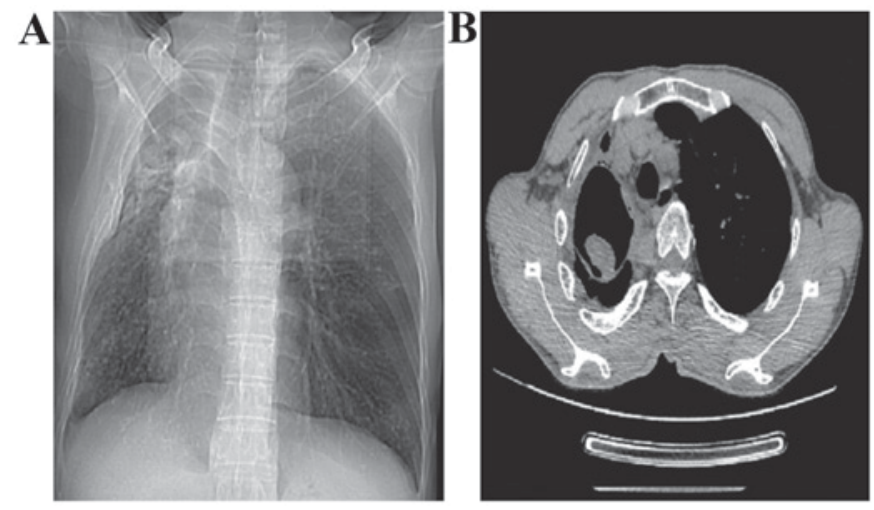

Figure 1. CT imaging of representative 'meniscus sign' of one pulmonary aspergilloma patient. (A) Coronal CT and (B) cross-sectional images.
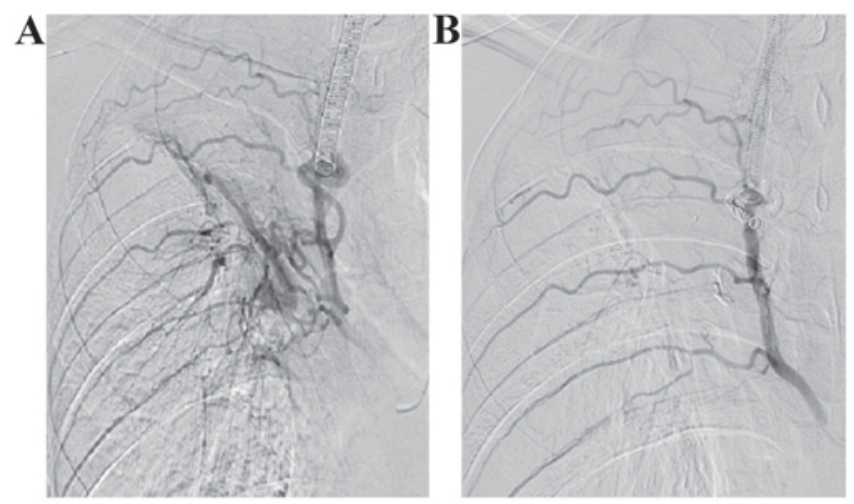

Figure 2. Angiography of intercostal and bronchial arteries. (A) Multiple intercostal and bronchial arteries from one common stem. (B) Combined embolism with PVA particle (diameter, 500-700 $\mu \mathrm{m}$ ), spring coil and NBCA glue, reserving intercostal arteries. PVA, polyvinyl alcohol; NBCA, $N$-butyl cyanoacrylate.

Surgical method. Under local anesthesia, Seldinger technology was used to puncture femoral artery at one side. Subsequently, we placed $4 \mathrm{~F}$ arterial sheath, selected the proper catheter (Cobra, Simmon) to perform bronchial arteriesa imaging and then observed for signs of increasing or expanding of bronchial arteries and concentration or leakage of contrast medium (Yangze Pharma, Taizhou, China). After defining the offending artery, $2.7 \mathrm{~F}$ microcatheter was chosen to perform super-selective catheterization and to perform embolotherapy over ostium of intercostal artery. Polyvinyl alcohol (PVA) particle (Yangze Pharma) was chosen as a common embolic agent (diameter, 500-700 $\mu \mathrm{m}$ ) and injected using low-pressure flow control method to avoid embolus backflow. If combined with obvious pulmonary artery fistula, the larger diameter gelatin sponge sections were used and PVA particulate embolization was performed. For obviously expanded bronchial arteries, if PVA particulate embolization was not ideal, then spring coil or $N$-butyl cyanoacrylate (NBCA) glue was used to continue embolism (Fig. 2) until the blood flow of the offending artery was retarded or embolism was arrested after contrast media flow back. Following digital subtraction angiography (DSA) radiography, the embolism was considered successful when abnormal staining at distant end disappeared. Since there are a large amount of bronchial arteries, all of them were observed carefully one by one. Besides observing
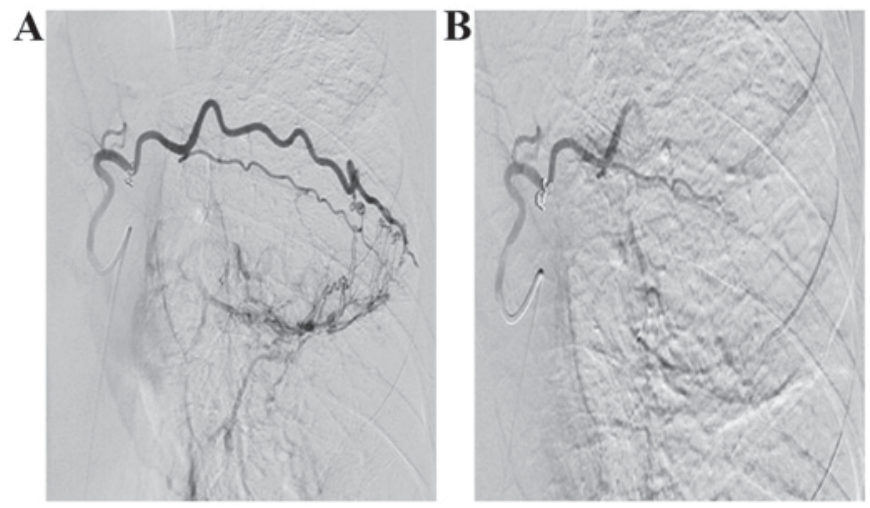

Figure 3. Angiography of left intercostal artery. (A) Arteriovenous malformation at distant end of left intercostal artery. (B) After PVA particle (diameter, 500-700 $\mu \mathrm{m}$ ) with spring coil embolism. PVA, polyvinyl alcohol.
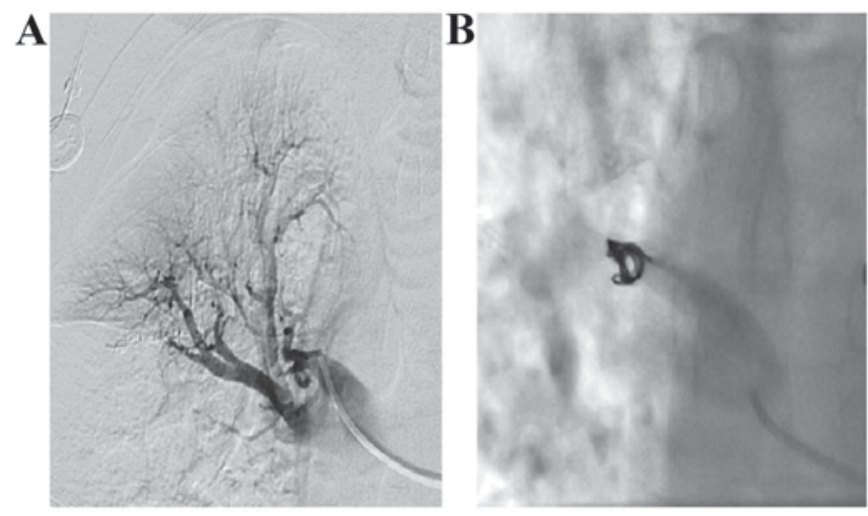

Figure 4. Angiography of right pulmonary artery and its branches. (A) Changes of blood flow direction of right pulmonary arterial branch. (B) Pulmonary artery after spring coil embolism.

bronchial arteries, the intercostal artery (Fig. 3), internal thoracic artery, inferior phrenic artery and pulmonary artery need to be checked for (Fig. 4) sources of bleeding. In addition, spinal arteries or its communicating branches need to be avoided during embolism. When removing the sheath after surgery, the puncture point is pressed for 15-20 min to stop bleeding with compression bandage, and then patient conditions were observed carefully for 2 weeks on anti-fungal therapy to avoid relapse.

\section{Results}

Through radiography, 68 embolism offending arteries were found, among 36 bronchial arteries (52.94\%), 15 intercostal arteries (22.06\%), 9 internal thoracic arteries (16.17\%), 5 inferior phrenic arteries (7.35\%), and 3 pulmonary arterial branches $(4.41 \%)$. Vital signs of 25 patients after surgery were stable with no death during perioperative period or complications such as spinal cord injury, intercostal cutaneous necrosis, substernal burning sensation or bronchoesophageal fistula.

Massive hemoptysis of 21 cases stopped after embolotherapy immediately and the effective rate of embolism was $84.00 \%$; for 3 cases, massive hemoptysis occured within $24 \mathrm{~h}$ and it was stopped through second imaging with embolism; 
1 patient had a small amount of hemoptysis after surgery and it was stopped after corresponding medical treatment; in addition, 10 cases were treated with PVA particulate embolization solely, 12 cases with PVA with spring coil and 3 cases with PVA, spring coil, and NBCA glue.

\section{Discussion}

Pulmonary aspergillosis is closely related to basic diseases of lung, most cases are secondary to diseases such as pulmonary tuberculosis, bronchiectasia, pulmonary cystic fibrosis, pulmonary cyst, and lung cancer and it is reported that the ratio of cases secondary to pulmonary tuberculosis is $80.2 \%$ (4). Aspergillus parasiticus is easily formed on the upper respiratory tract and diseases are caused when organism immunity is low. Aspergillosis can multiply within cavity and characteristic aspergilloma will be formed with a large amount of mycelium mixing with bleeding sphacelus, fibrous protein, mucosa, epithelial cell and inflammatory necrosis cells. Besides purulent hemorrhagic inflammation of inflammatory granulation tissue, aspergilloma will also cause necrosis and bleeding of tissues and vessels due to its erosion of blood vessels by active enzyme toxins; in addition, rich vasoganglion even arterial aneurysm will be formed due to inflammatory stimulation around the lesion, its mechanical motion will invade intercostal and bronchus arteries to cause angiorrhexis and massive hemoptysis with life-threathening risks (5). There are great difficulties for medical treatment of massive hemoptysis, which has no obvious effects; surgical removal provides a radical treatment method, which is considered as the first option $(6,7)$, but most old patients are also suffering from lung diseases such as pulmonary tuberculosis, pneumoconiosis, and bronchiectasia, the lung function has decreased severely so the risks of surgery are greatly increased.

Bronchial artery embolization (BAE) was first suggested in 1974 and it has become the most effective method of controlling massive hemoptysis currently through 40 years of developing and improving, which is featured by being safe, minimally invasive and highly efficient and its success rate of hemostasis is more than $90 \%$ (8). In our cases embolism was performed in 25 cases, of these hemoptysis in 21 cases was stopped immediately and the effective rate was $84.00 \%$, and it basically agrees with other reports. In order to improve the successful rate of BAE, it is believed by us that the following items should be paid attention to during surgery: i) Finding of all offending arteries. The main resource is bronchial artery and the opening is at the thoracic anterior aortic wall or side wall of 5 th to 6 th thoracic vertebrae, in most cases, there are 1-2 on the right and 2-4 on the left. About 1/3 cases have bronchial arteries on both sides from one stem and when the catheter is passing through, there is a hooking sensation. For a small number of cases, it is from internal thoracic artery, intercostal artery, subclavian artery, phrenicartery and thyrocervical trunk. In order to improve the successful rate of embolism, chest CT scanning is to be performed before surgery to determine the bleeding location within lung and the surroundings. All offending arteries need to be found according to lesion location. If the lesion is at lobus inferior pulmonis, then phrenicartery needs to be located, if the lesion is at anterior upper lung lobe, then subclavian artery, internal thoracic artery, and axillary artery are targets. Corresponding intercostal arteries shall be considered if there is local pleural thickening. If the above-mentioned systemic circulation branches are normal, then it may be due to pulmonary artery bleeding. In this study, 1 case had massive hemoptysis within $24 \mathrm{~h}$ after surgery, and abnormal pulmonary artery was found during 2nd imaging and it was stopped through another embolotherapy of massive hemoptysis. Therefore, chest CTA before surgery can well define the offending artery, which will save surgery time and greatly improve the success rate of surgery. ii) Choosing proper embolic agent. PVA particle and spring coil are common embolic agents, the first one is cheap and permanent nonantigenic embolic agent, the common diameter is $500-700 \mu \mathrm{m}$, the embolism will be incomplete if the diameter is too large, and it will cause injuries to vascular bed of arteries if the diameter is too small. After re-examination of DSA, gelatin sponge pieces, spring coil or NBCA glue can be added if the blood flow is too fast for accurate location and permanent hemostasis. It has been revealed in study that there are different diameters of embolic agent, compact embolism is able to prevent bleeding arteries from establishing collateral circulation, and effects of combined embolism with more than 2 groups are better than one single embolic agent (9). iii) Avoiding spinal cord injury and ectopic embolism. The most severe complication of BAE is spinal cord injury and its incidence rate is $2-3 \%$, and its main manifestations are transverse sensation and dyskinesia, once it occurs, hormone, vascular dilation and neuro-nutrition drug treatments should be positively performed. Most patients will partially or completely recover within weeks or months, but some patients may have permanent injuries. After bronchial artery radiography, the results need to be read carefully, superselective microcatheter is used when there is hairpin running anterior spinal artery; PVA particulate with diameter $>500 \mu \mathrm{m}$ is used in embolization to avoid embolism of anterior spinal artery. In addition, embolism process needs to be gentle to avoid ectopic embolism outside the offending arteries caused by fast injection rate of embolic agent and large pressure. There are no severe complications for patients in this group, and it is considered that it may be related to careful angiography, superselective catheterization and application of microcatheter before embolism. No special treatments are needed generally for other complications such as low-grade fever, intercostal pain, substernal burning sensation, or dysphagia.

Above all, for patients who suffer from massive hemoptysis of pulmonary aspergilloma and their medical treatments are not effective and they have no surgery conditions, bronchial arteries embolism technique is a simple and effective method as salvage hemostasis method. Good understanding of the indications and complete and thorough embolism of bleeding arteries is the key of successful surgery.

\section{References}

1. Brown HA, Aruny JE, Elefteriades JA and Sumpio BE: Subclavian aneurysm presenting with massive hemoptysis: a case report and review of the literature. Int J Angiol 22: 69-74, 2013.

2. Babatasi G, Massetti M, Chapelier A, Fadel E, Macchiarini P, Khayat A and Dartevelle P: Surgical treatment of pulmonary aspergilloma: Current outcome. J Thorac Cardiovasc Surg 119: 906-912, 2000 
3. Aydoğdu K, İncekara F, Sahin MF, Gülhan SS, Findik G, Taştepe İ and Kaya S: Surgical management of pulmonary aspergilloma: clinical experience with 77 cases. Turk J Med Sci 45: 431-437, 2015

4. Lee JG, Lee CY, Park IK, Kim DJ, Chang J, Kim SK and Chung KY: Pulmonary aspergilloma: analysis of prognosis in relation to symptoms and treatment. J Thorac Cardiovasc Surg 138: 820-825, 2009

5. Muniappan A, Tapias LF, Butala P, Wain JC, Wright CD, Donahue DM, Gaissert HA, Lanuti M and Mathisen DJ: Surgical therapy of pulmonary aspergillomas: a 30-year North American experience. Ann Thorac Surg 97: 432-438, 2014.

6. Ichinose J, Kohno T and Fujimori S: Video-assisted thoracic surgery for pulmonary aspergilloma. Interact Cardiovasc Thorac Surg 10: 927-930, 2010
7. Gezer S, Tastepe I, Sirmali M, Findik G, Türüt H, Kaya S, Karaoğlanoğlu N and Cetin G: Pulmonary sequestration: a single-institutional series composed of 27 cases. J Thorac Cardiovasc Surg 133: 955-959, 2007.

8. Alexander GR: A retrospective review comparing the treatment outcomes of emergency lung resection for massive haemoptysis with and without preoperative bronchial artery embolization. Eur J Cardiothorac Surg 45: 251-255, 2014

9. Lorenz J, Sheth D and Patel J: Bronchial artery embolization. Semin Intervent Radiol 29: 155-160, 2012. 